\title{
A Retroprospective Study of an Antimicrobial Stewardship Program on Antimicrobial Utilization at a Tertiary Care Medical Center in Kolkata
}

\section{Dr Sujay Ranjan Deb ${ }^{1}$, Dr Sourav Maiti ${ }^{2}$, Dr Arpan Dutta Roy ${ }^{3}$, Dr Prolay Paul ${ }^{4}$, Dipankar Maiti ${ }^{5^{*}}$, Triyasha Adhikary ${ }^{5}$}

1. Dy. Medical Director, Ruby General Hospital, Kolkata, India.

2. MD DNB MNAMS, Consultant Microbiologist \& Infection Control Officer, Ruby General Hospital, Kolkata, India.

3. Chief of Clinical Pharmacology, Certified NABH Assessor (QCI) HOPE, Doctor of Pharmacy (PB), (PhD.) Pharmacology, Ruby General Hospital, Kolkata, India.

4. Clinical Pharmacologist, Narayana Superspeciality Hospital, Howrah, West Bengal, India.

5. Final Year B.Sc. Nursing, Adichunchanagiri College of Nursing, Rajiv Gandhi University of Health Sciences, Bangalore, Karnataka, India.

\author{
*Corresponding author's E-mail: dipankarmaiti2015@gmail.com
}

Received: 14-07-2021; Revised: 19-09-2021; Accepted: 25-09-2021; Published on: 15-10-2021.

\begin{abstract}
The WHO has set Defined Daily Dose which represent the average daily dose of an antibiotic in a standard patient. The DDD mainly focuses on population-based parameters \& assumes that patients as well as hospitals are homogenous entities. DOTs are very useful in order to classify antibiotic days based on patient-level exposure. DOTs merely mean the number of days that a patient is on an antibiotic, irrespective of dose. DOTs signifies that the underlying assumptions about antibiotic dosing was appropriate. Additionally, when patients receive more than one antibiotic, supplementary DOT may be counted. The 300-bed tertiary care medical center serves adults and paediatrics. An all-time Microbiology Consultant and a Clinical Pharmacology trainee used to go for round daily and used to collect data for ASP for the period of 3 months that is April to June,2021. In this study we have compared DOT of some important antibiotics for a specific period of time for both COVID and NON COVID patient. ASP-focused antibiotics were antibiotics routinely evaluated by the ASP team for appropriateness during therapy and the potential to optimize their appropriate use through policies, protocols, formulary restrictions, or clinician education. ASP-focused antibiotics included meropenem, linezolid, pip-taz, poly b, colistin, teicoplanin. In this study we have compared the DDD for 2 specific period of time for better understanding the consumption of those antibiotics. In conclusion, following the initiation of an ASP, significant decreases in utilization, increases in cost savings occurred. In our study we have reduced the consumption and DDD of linezolid which is clinically significant. When it comes to DOTs; We have reduced the DOTs of piptaz and teicoplanin for covid patient And Reduced the DOTs of meropenem and teicoplanin for noncovid patient which is clinically and statistically significant.
\end{abstract}

Keywords: Antibiotic Stewardship, Antimicrobials, Days of Therapy, Defined Daily Dose, Teicoplanin.

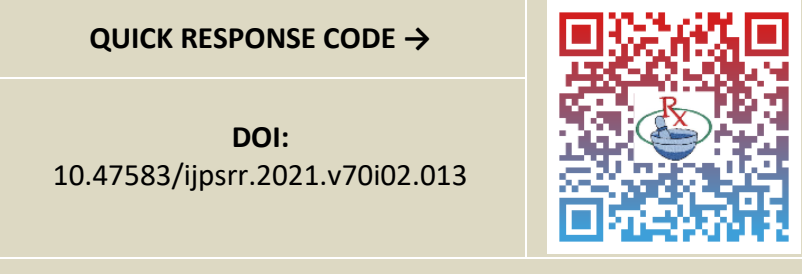

DOI link: http://dx.doi.org/10.47583/ijpsrr.2021.v70i02.013

\section{INTRODUCTION}

D ue to Increased antibiotic resistance combined with minimal market introduction of new classes of antibiotics results to a multitude of initiatives to increase awareness and institute solutions to address the problem coined as "bad bugs, no drugs." ${ }^{1}$ The Infectious Disease Society of America has released as well as promoted the $10 \times 20$ initiative, which seeks to achieve 10 new antibacterial drug approvals by the US Food and Drug Administration by 2020 . - Simultaneously the IDSA and the SHEA together had released antimicrobial stewardship guidelines outlining the importance of antimicrobial stewardship programs. ${ }^{3}$ In the year 2013, a publication from the Centers for Disease Control and Prevention gave notice about antimicrobial resistance issues. ${ }^{4} \mathrm{Dr}$. Tom Frieden, The CDC's director, reacted about this issue of antimicrobial resistance at a press conference, saying, "If we are not careful, we will soon be in a post-antibiotic era. And, in fact, for some patients and some microbes, we are already there." $\underline{5}$ These notices of Dr. Tom Frieden were drawn the attention on a national level on September 18, 2014, with the signing of the executive order Combating Antibiotic Resistant Bacteria by the former President Barack Obama \& established the Task Force for Combating Antibiotic-Resistant Bacteria. $\underline{6}$ Subsequently, the Task Force was assembled in March of 2015 \& released a national action plan. - The White House accommodated a National Antibiotic Stewardship Setting in the month of June, 2015 that carried out together key shareholders in the field to make assurances to combat antibiotic resistance. $\stackrel{8}{ }$ Latterly, on September 15, 2015, the US Department of Health and Human Services, the US Department of Agriculture \& the US Department of Defense chosen human and animal health specialists to the Presidential Advisory Council on Protesting AntibioticResistant Bacteria such that recommendations can be 
made about the issues of preserving current antibiotics \& decreasing the uprising of resistance. $\frac{9}{}$

Antimicrobial stewardship programs (ASPs) have been manifested to impact the emergence of antimicrobialresistant bacteria. ${ }^{8}$ Simultaneously to achieve a reduction of antimicrobial governance, Antimicrobial stewardship programs (ASPs) reduce health care financial expenditures with improve patient outcomes and safety. Thus, patients are in safer side in this aspect. ${ }^{10}$ The clinical outcomes of ASP possess significant challenges in measurement and evaluation of antimicrobial resistance. They have given enough evidence based on their research aspect. In one hand, measures of antimicrobial utilization $\&$ in other hand financial expenditures are established and accessible outcomes for demonstrating the value contributed by ASPs. The upshot of stewardship programs about cost avoidance as well as decreased antibiotic utilization is well described.${ }^{10}$ As there are obvious limits to cost reduction or avoidance, the need to evaluate other important stewardship outcomes measures, including patient outcomes such as rate of cure, length of stay, or antibiotic resistance changes, side effects of the drug in patients' body is increasingly important \& costs savings may be used continually to justify ongoing support for ASPs. Though the existence of such programs will soon be compulsary, ${ }^{6,11}$ the scope \& size may vary with their efficacy in meeting basic aims of optimizing anti-infective utilization as well as patient outcomes. The objective of this study was to point out simultaneously the effect of the program about antimicrobial utilization \& related expenditures to determine any impact on antibiotic resistance. $^{9}$

Antimicrobial consumption is a clustered rate of antimicrobial used for benchmarking purposes. To determine the rate, a simple proportion is constructed with the numerator as amount utilized and the denominator as patients eligible for antimicrobials within a system. ${ }^{3}$ The couple of main measures for amount are generally accepted, days of therapy \& defined daily dose. The WHO has set Defined Daily Dose which represent the average daily dose of an antibiotic in a standard patient. The DDD mainly focuses on population-based parameters \& assumes that patients as well as hospitals are homogenous entities. For example, compared to Normal Renal Functioned people will a Renal Impaired patient will receive a fewer doses \& amounts of antibiotics to achieve the same pharmacokinetics. DOTs are very useful in order to classify antibiotic days based on patient-level exposure. DOTs merely mean the number of days that a patient is on an antibiotic, irrespective of dose. However, this system also having some limitations. DOTs signifies that the underlying assumptions about antibiotic dosing was appropriate. Additionally, when patients receive more than one antibiotic, supplementary DOT may be counted. This may generate problem if endeavoring to classify a composite end point of total antibiotic use. The abovementioned methods are standardized with a denominator such as hospitalized patient days, and 1000 patient days is the convention. As both strategies have limitations the results can't be correlate always. An example of our own results give support to this point. When there are exceptions to the correlation, rates should be compared in case the method used is constant. ${ }^{11}$

\section{METHODOLOGY}

The 300-bed tertiary care medical center serves adults and paediatrics. The program focuses on improving the use of antimicrobials in the adult inpatient population through a variety of preauthorization (front end) and prospective audit and feedback (back end) initiatives, respectively. Additionally, preparation and implementation of empiric therapy guidelines, clinical pathways, and clinician education are used to pursue the program's goals. An alltime Microbiology Consultant and a Clinical Pharmacology trainee used to go for round daily and used to collect data for ASP for the period of 3 months that is April to June, 2021. In this study we have compared DOT of some important antibiotics for a specific period of time for both COVID and NON COVID patient. ASP-focused antibiotics were antibiotics routinely evaluated by the ASP team for appropriateness during therapy and the potential to optimize their appropriate use through policies, protocols, formulary restrictions, or clinician education. ASP-focused antibiotics included meropenem, linezolid, pip-taz, poly b, colistin, teicoplanin. In this study we have compared the DDD for 2 specific period of time for better understanding the consumption of those antibiotics.

Inclusion criteria: All age gender admitted in critical care unit included in the study.

Exclusion criteria: Patient visiting Outpatient are excluded from the study.

\section{Data Collection and Analysis}

Utilization data for antimicrobials were obtained for JANUARY to JUNE,2021 from a previously created database that was established using the hospital pharmacy computer system and transferring the data to a spreadsheet (Microsoft Excel) with subsequent data deidentification. Medication orders were summed per medication per quarter in grams using the total number of doses and dose per administration. The grams were then converted to census-normalized DDD using World Health Organization (WHO) Totals for all ASP-focused and all systemic antibiotics were calculated per quarter. Changes in utilization were evaluated for the specific period of time before and after the ASP implementation. Antiviral agent data were excluded from all analyses. The list of organisms evaluated included Pseudomonas aeruginosa, Escherichia coli, Klebsiella pneumoniae, Enterobacter cloacae, Serratia marcescens, Acinetobacter baumannii, Staphylococcus aureus, coagulase-negative staphylococci, Enterococcus faecalis, Enterococcus faecium, and Streptococcus pneumoniae. Changes in susceptibility were evaluated for ASP-focused antibiotics only. No changes in interpretative susceptibility testing were adopted during this period. 
Medcalc statistical software was utilized for data analysis of DOTs.

\section{RESULTS}

Notable significant findings in utilization between the periods included a decrease in piptaz DOTs $(p=.0237)$ and a decrease in teicoplanin DOTs $(p=.0006)$ for covid patient. Another notable significant finding in utilization between the periods included a decrease in meropenem DOTs $(p=.0001)$ and decrease in teicoplanin DOTs $(p<$ .0001) for Non covid patient.

Table 1: DOTs in days for Covid patients (Period 1= April to May,2021 and Period 2= June,2021)

\begin{tabular}{|l|c|c|c|}
\hline Antibiotics & $\begin{array}{c}\text { Period } \\
\mathbf{1}(\mathbf{N}=\mathbf{2 3}) \\
\text { (Mean } \pm \text { SD) }\end{array}$ & $\begin{array}{c}\text { Period } \\
\mathbf{2 ( N = 2 6 )} \\
\text { (Mean } \pm \text { SD) }\end{array}$ & P Value \\
\hline Meropenem & $5.4 \pm 3.5$ & $5.5 \pm 3.2$ & 0.9172 \\
\hline Piptaz & $4.0 \pm 1.6$ & $2.7 \pm 2.2$ & 0.0237 \\
\hline Teicoplanin & $6.5 \pm 2.1$ & $4.5 \pm 1.7$ & 0.0006 \\
\hline Doxycycline & $5.4 \pm 2.4$ & $4.1 \pm 3.0$ & 0.1035 \\
\hline
\end{tabular}

Table 2: DOTs in days for Non Covid patients (Period $1=$ April to May,2021 and Period 2= June,2021)

\begin{tabular}{|c|c|c|c|}
\hline Antibiotics & $\begin{array}{c}\text { Period } \\
1(\mathrm{~N}=18) \\
\text { (Mean } \pm S D)\end{array}$ & $\begin{array}{c}\text { Period } \\
2(\mathrm{~N}=15) \\
\text { (Mean士SD) }\end{array}$ & P Value \\
\hline Meropenem & $6.8 \pm 3.1$ & $2.6 \pm 1.8$ & 0.0001 \\
\hline Piptaz & $2.8 \pm 1.5$ & $3.0 \pm 2.0$ & 0.7523 \\
\hline Teicoplanin & $5.0 \pm 1.4$ & $2.0 \pm 1.7$ & $<0.0001$ \\
\hline Metronidazole & $5.7 \pm 3.1$ & $5.0 \pm 1.4$ & 0.4548 \\
\hline
\end{tabular}

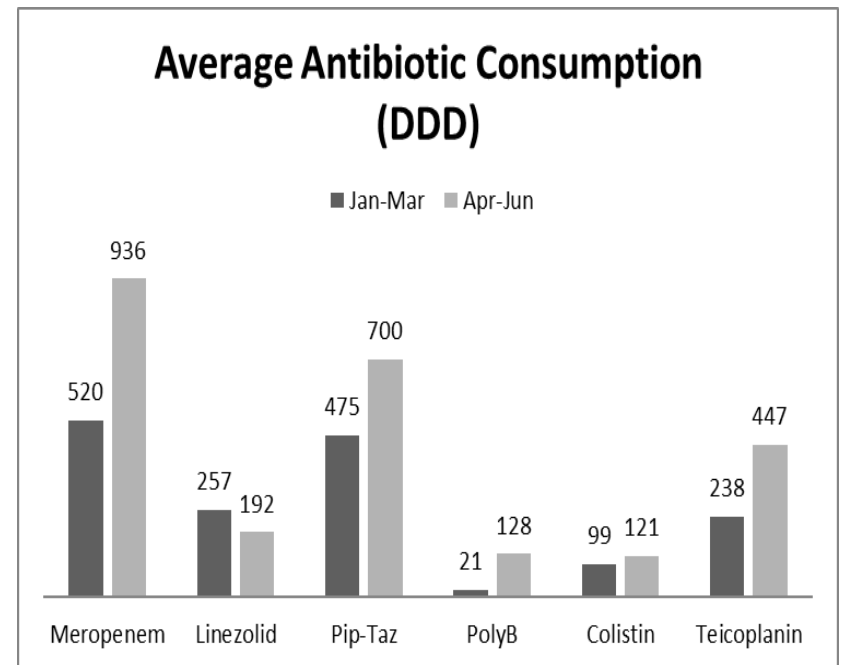

Figure 1: Average Antibiotic Consumption

Notable significant findings in DDD were found which include a decrease in linezolid use and increase in meropenem, piptaz, poly b, colistin and teicoplanin use.

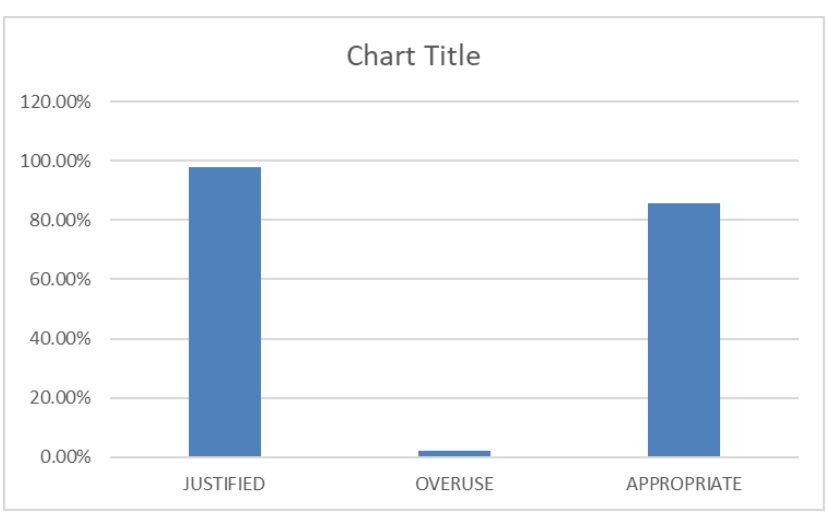

Figure 2: Appropriateness of Antimicrobial use During Antimicrobial stewardship program for covid patient

A significant finding was found in this ASP study around $85.71 \%$ prescription antibiotics were appropriate and $2.04 \%$ antibiotics were overused for covid patients.

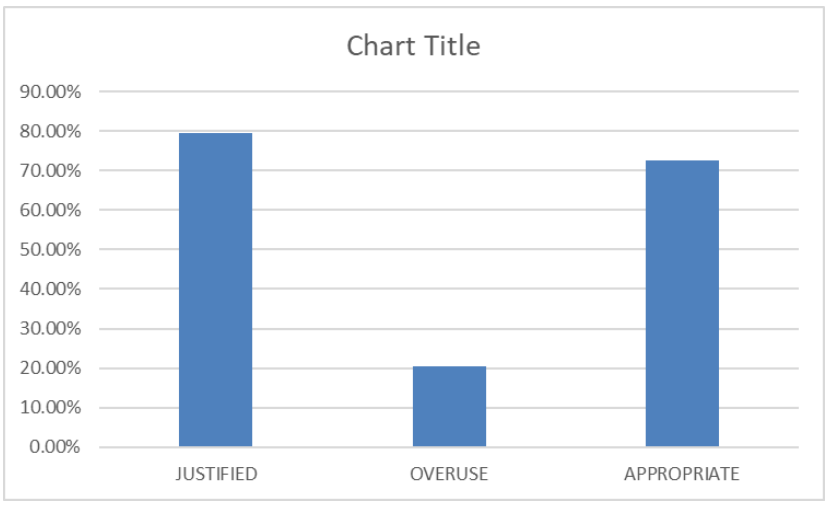

Figure 3: Appropriateness of Antimicrobial use During Antimicrobial stewardship program for Non covid patient

A significant finding was found in this study around $72.73 \%$ prescription antibiotics were appropriate and 20.51\% antibiotics were overused for Non covid patients.

\section{DISCUSSION}

This study aimed to characterize changes in antibioticutilization patterns and temporally related changes in bacterial susceptibility and drug expenditures that occurred in our institution following the introduction of an ASP. Observed changes included alterations in antibiotic utilization, antibiotic financial expenditures, and bacterial susceptibilities. ASP interventions designed to achieve these changes included formulary restrictions, prospective audits and feedback, dose optimizations, intravenous to oral conversions, development of empiric therapy guidelines and clinical pathways, and clinician education. Following the introduction of the ASP, mean ASP-focused antibiotic utilization decreased. This is similar to results from other studies evaluated in a meta-analysis that showed $11 \%$ to $38 \%$ reduction in DDD per 1,000 patient days due to stewardship interventions. ${ }^{12}$

Further breakdown of the utilization reflected that piperacillin/tazobactam and teicoplanin use decreased for covid patient; meropenem and teicoplanin use decreased for Non covid patient. These findings further support our 
observed qualitative changes in antibiotic use, which translated into financial savings. The relationship between antibiotic use and susceptibility rates has been inconsistent in a variety of studies. ${ }^{13,14}$ ASP periods, a decrease in overall linezolid use was observed.

\section{Limitation}

This study is not without limitations. While ASP outcomes of resistance, antibiotic utilization were evaluated, important ASP clinical goals of improving the safe and efficacious use of antibiotics for improving patient outcomes were not. Use of Doxycycline in covid patient is under the protocol of covid treatment so we have not analyzed the use of Doxycycline for the same. Due to unavailability of covid patient in the month of April we have started taking covid data from may and continued till June.

\section{CONCLUSION}

In conclusion, following the initiation of an ASP, significant decreases in utilization, increases in cost savings occurred. In our study we have reduced the consumption and DDD of linezolid which is clinically significant. When it comes to DOTs; We have reduced the DOTs of piptaz and teicoplanin for covid patient And Reduced the DOTs of meropenem and teicoplanin for non-covid patient which is clinically and statistically significant. These outcomes are consistent with the goals of decreasing unnecessary antibiotic exposure to patients, decreasing rates of resistance, and reducing avoidable health care financial expenditures. Such findings are utilized in the ongoing justification for maintaining and expanding our stewardship program, and this evaluative exercise serves as an example that can be employed by other similar stewardship programs.

\section{List of abbreviation:}

DOT- Days of Therapy

DDD- Defined Daily Dose

ASP- Antimicrobial Stewardship Program

Pip-taz - Piperacillin- Tazobactam

CDC- Center for Disease Control

IDSA- Infectious Disease Society of America

SHEA- Society for Healthcare Epidemiology of America

\section{REFERENCES}

1. Boucher HW, Talbot GH, Bradley JS. et al. Bad bugs, no drugs: No ESKAPE! An update from the Infectious Diseases Society of America. Clin Infect Dis. 2009; 48(1): 1- 12. [PubMed] [Google Scholar]
2. The $10 \times$ '20 Initiative: Pursuing a global commitment to develop 10 new antibacterial drugs by 2020. Clin Infect Dis. 2010; 50( 8): 1081-1083. [PubMed] [Google Scholar]

3. Dellit TH, Owens RC, McGowan JEJ. et al. Infectious Diseases Society of America and the Society for Healthcare Epidemiology of America guidelines for developing an institutional program to enhance antimicrobial stewardship. Clin Infect Dis. 2007; 44(2): 159- 177. [PubMed] [Google Scholar]

4. Centers for Disease Control and Prevention (CDC). . Antibiotic resistance threats in the United States, 2013. http://www.cdc.gov/drugresistance/threat-report-2013/pdf/arthreats-2013-508.pdf. Accessed September 17, 2015.

5. Centers for Disease Control and Prevention (CDC). . CDC telebriefing on today's drug-resistant health threats. CDC Online Newsroom - Press Briefing

Transcript. http://www.cdc.gov/media/releases/2013/t0916 healththreats.html. Accessed February 1, 2014.

6. White House. . Executive order: Combating antibiotic-resistant bacteria. http://www.whitehouse.gov/the-pressoffice/2014/09/18/executive-ordercombating-antibiotic-resistantbacteria. Accessed September 17, 2015.

7. White House. . National action plan for combating-antibiotic-resistant bacteria. http://www.whitehouse.gov/sites/default/files/docs/national action plan for combating antibotic-resistant bacteria.pdf. Accessed September 17, 2015.

8. White House. . Over 150 animal and health stakeholders join White House effort to combat antibiotic resistance. https://www.whitehouse.gov/the-press-

office/2015/06/02/fact-sheet-over-150-animal-and-health-stakeholdersjoin-whitehouse-effo. Accessed September 21, 2015.

9. US Department of Health and Human Services. . Establishment of the Presidential Advisory Council on Combating Antibiotic-Resistant Bacteria. http://www.hhs.gov/about/news/2015/09/15/establishmentof-the-presidential-advisory-council-on-combating-antibiotic-resistantbacteria. Accessed November 9, 2015.

10. McGowan JE. Antimicrobial stewardship - the state of the art in 2011: Focus on outcome and methods. Infect Control Hosp Epidemiol. 2012; 33( 4): 331-337. [PubMed] [Google Scholar]

11. Federal Register. . Reform of requirements for long-term care facilities. https://www.federalregister.gov/articles/2015/07/16/2015$17207 /$ medicare-and-medicaid-programs-reform-of-requirements-forlong-term-care-facilities. Accessed March 5, 2016. [PubMed]

12. Kaki R, Elligsen M, Walker S, Simor A, Palmay L, Daneman N. Impact of antimicrobial stewardship in critical care: A systematic review.J Antimicrob Chemother. 2011; 66(6): 1223- 1230. [PubMed] [Google Scholar]

13. Bosso JA. The impact of antibiotic management on resistance. Pharmacotherapy. 2004; 24(12 Pt 2): 224S- 31S. [PubMed] [Google Scholar]

14. Schuts EC, Hulscher MEJL, Mouton JW. et al. Current evidence on hospital antimicrobial stewardship objectives: A systematic review and meta-analysis [published online ahead of print March 2, 2016]. Lancet Infect Dis. [PubMed] [Google Scholar]

Source of Support: The author(s) received no financial support for the research, authorship, and/or publication of this article.

Conflict of Interest: The author(s) declared no potential conflicts of interest with respect to the research, authorship, and/or publication of this article.

For any question relates to this article, please reach us at: editor@globalresearchonline.net New manuscripts for publication can be submitted at: submit@globalresearchonline.net and submit_ijpsrr@rediffmail.com 